Physical Sciences | Akihiro Morita

\section{Facilitated ion transfer through liquid-liquid interfaces}

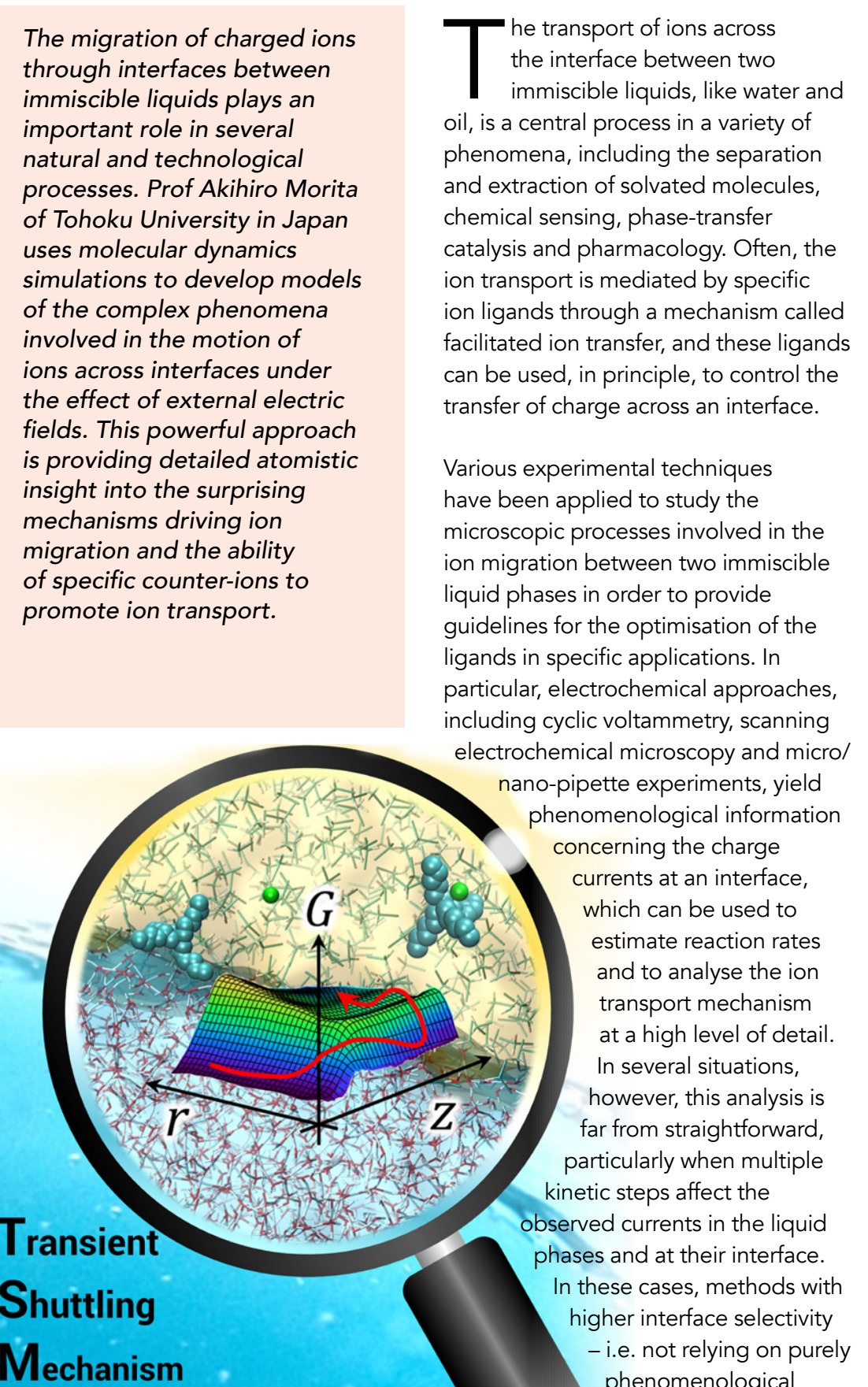

Information - are needed to arrive at a reliable model of the interfacial transfer inetics and mechanism.

Another challenge in the study determination of their microscopic structure, whose knowledge is of crucial importance in understanding ion transfer mechanisms. A limited number of experimental techniques, like sum frequency generation and $\mathrm{X}$-ray spectroscopy, are capable of providing structural information for interfaces between different classes of liquids, but the study of structural fluctuations remains extremely challenging with these methods alone. To overcome these limitations, Prof Akihiro Morita of Tohoku University in Japan has been developing computer simulation techniques that provide an atomistic picture of the structure of liquid-liquid preffaces and allow one to follow the has been used to gain unprecedented insight not only into the microscopic mechanism of ion transport and on the structure and dynamics of various liquid-liquid interfaces, but also into the effect of different ligands on the transport kinetics.

\section{MOLECULAR DYNAMICS}

AT LIOUID-LIOUID INTERFACES Molecular dynamics is a class of simulation techniques that provide a real-time picture of how atoms and molecules evolve for a particular set of thermodynamic conditions, such as temperature, density, pressure and, potentially, the presence of an external via a force field (which is, in essence, a mathematical model of how atoms se poch other), which in turn affects their evolves in time. This makes it possible not only to identify potential competing mechanisms of ion transport, but also to estimate macroscopic properties, including kinetic constants and diffusion coefficients which describe how fast ions move at an interface. This approach can therefore be used to complement and aid the interpretation of experimental measurements. Prof Morita and his collaborators have been pioneering the application of molecular dynamics techniques to liquid interfaces, and they have developed a
set of powerful methods to analyse molecular dynamics trajectories, elucidate the catalytic mechanism of ion transport at an inte

\section{MULTIDIMENSIONAL} FREE ENERGY SURFACES

One of the limitations of stand molecular dynamics simulations is their inability to treat systems containing many atoms for times long enough to detect phenomena like ion diffusion especially when the mechanism of transport involves one or more energy barriers. To solve this problem in the case of ion migration at liquid interfaces, Prof Morita's team have developed methods based on the calculation of tree energies from molecular dynamies trajectines. This approach involves ion migration, which may not be limited to a simple linear diffusion path along the membrane and can therefore have multiple spatial components. This

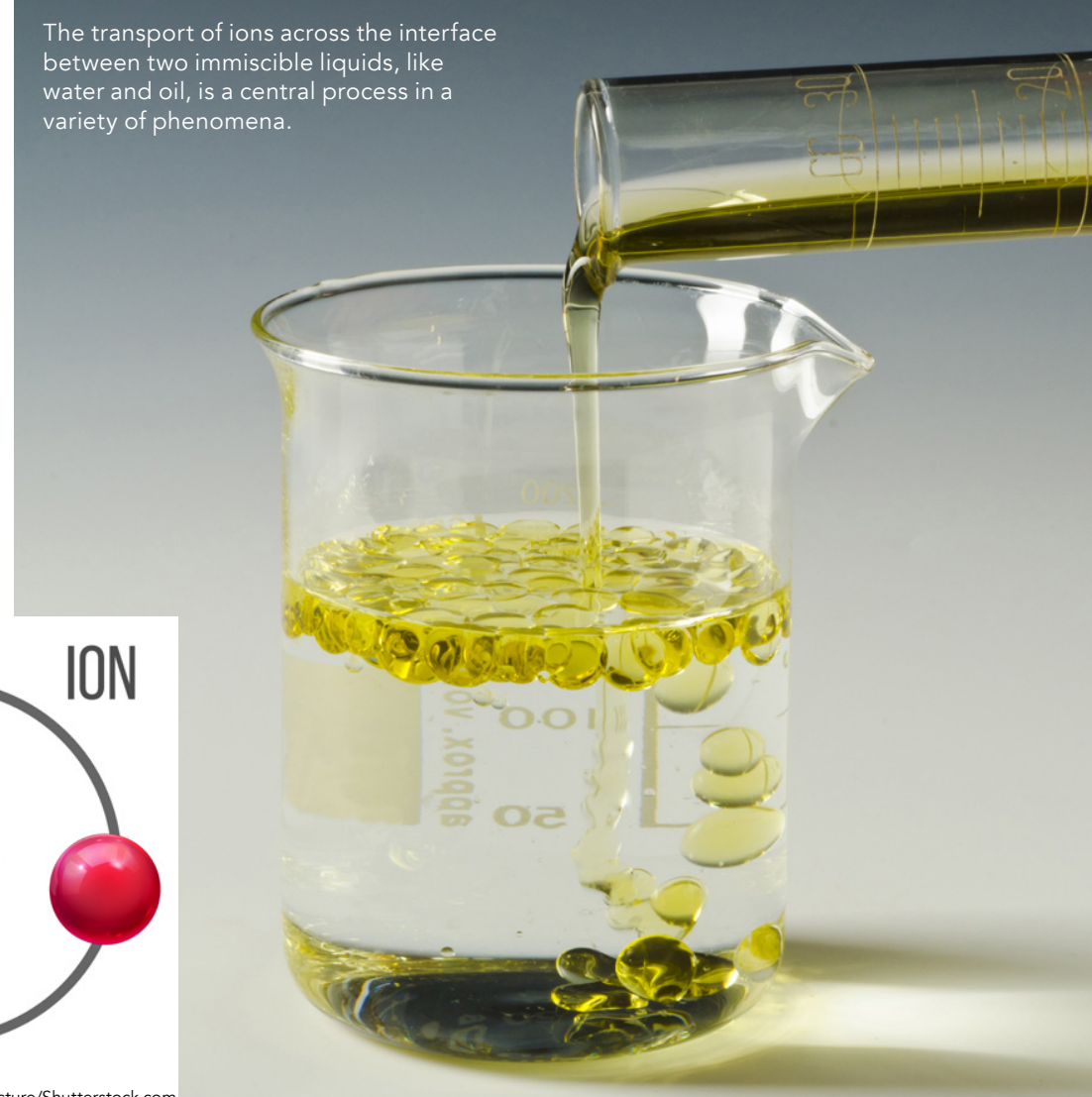

Molecular dynamics simulations provide details of the structure and dynamics of liquid-liquid interfaces at the level of single atoms and molecules.

multidimensional free energy profil at a given temperature, from which

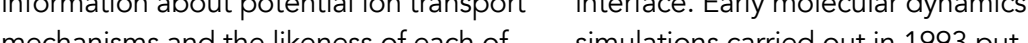
these mechanisms can be assessed.

\section{WATER-OIL INTERFACES:}

\section{THE WATER FINGER}

An interesting application of this approach has been in the study of simulations carried out in 1993 put 'water finger' - a trail of water molecules that accompany $\mathrm{Cl}$ as this ion moves However, these simulations were unable to provide estimates of ion transfer rates

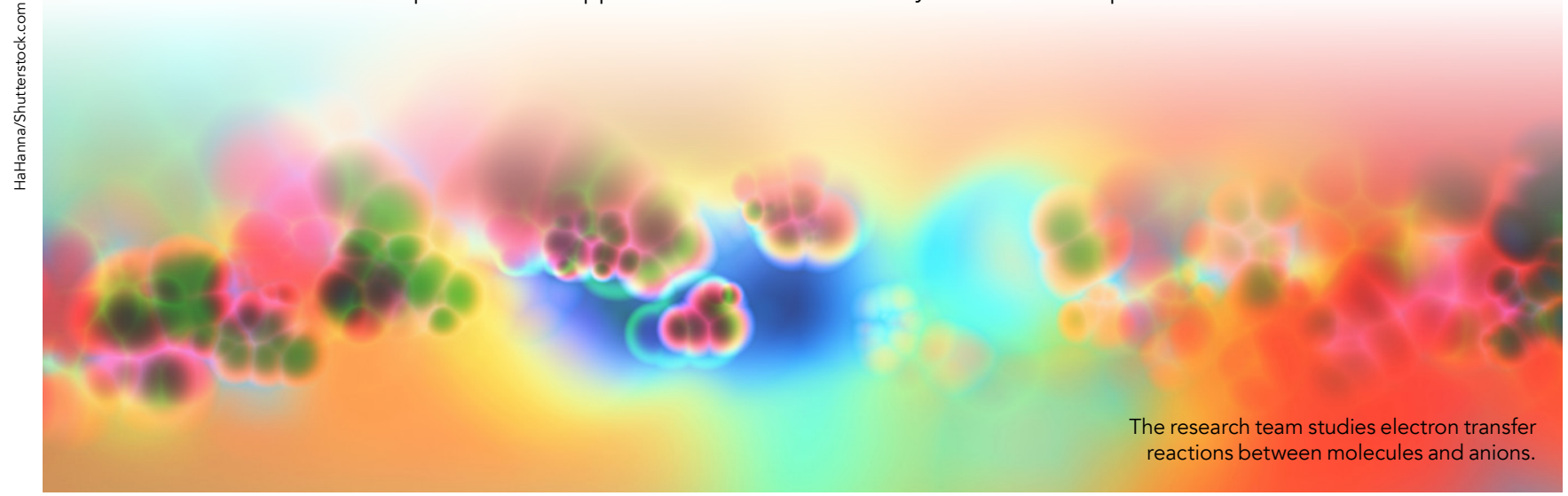




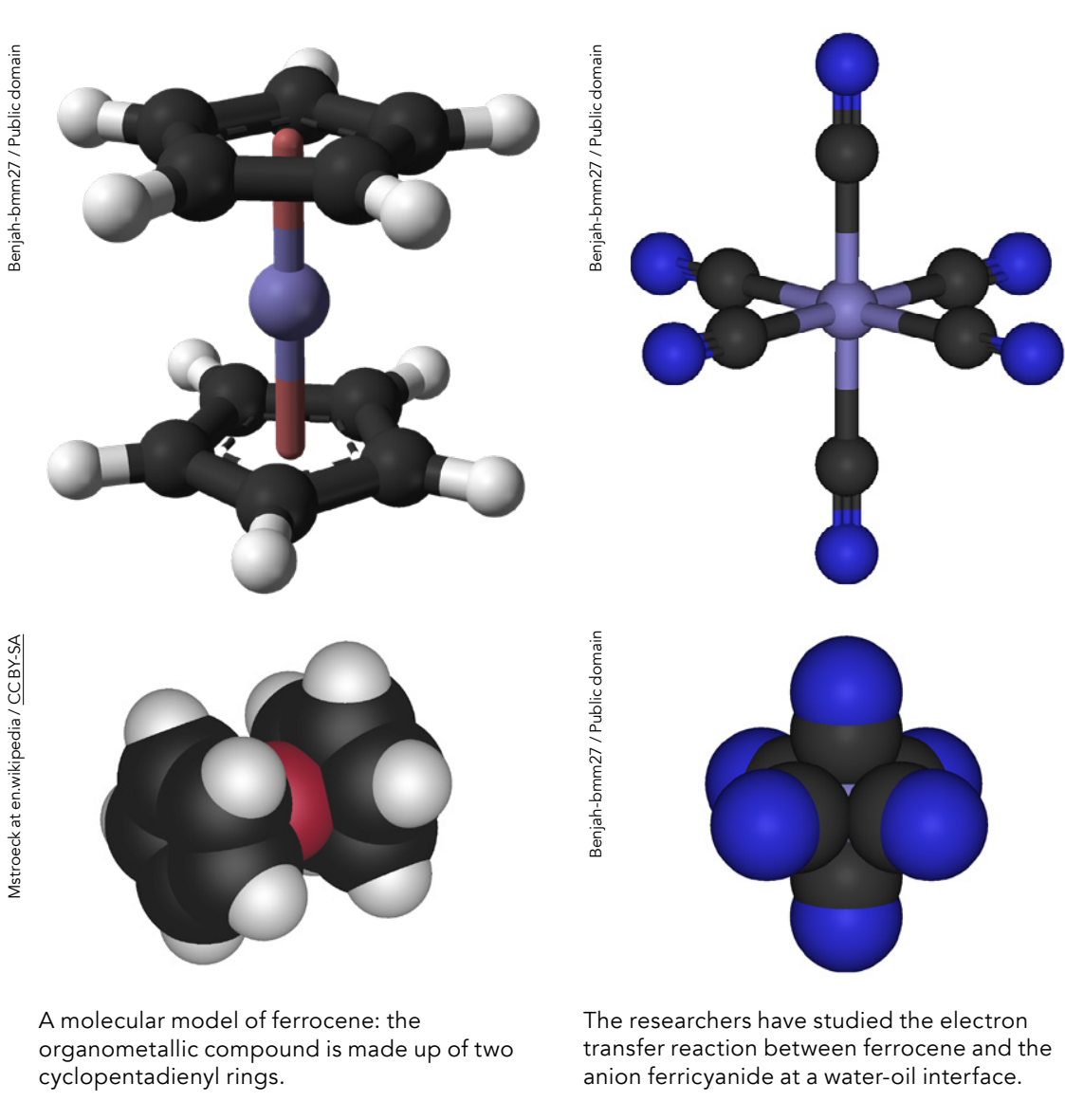

The formation of transient complexes involving hydrophobic ligands promotes the transport of hard hydrophilic ions across water-oil interfaces.

close to those measured in experiment, which was suggestive of a deviation of behaviour observed in the simulations.

Using their multidimensional free energy approach, the researchers were able to show that the mechanism of $\mathrm{C}$ - transport does indeed involve a hidden barrier for the ion's migration through the hydrophobic phase, which is inconsiste with simple diffusion. Even more interestingly, they demonstrated that this barrier originates from the formation and subsequent breaking of the water finger, thus reconciling the simulated behaviou and the experimentally observed migration rates.

\section{ELECTRON TRANSFER AT}

THE WATER-OIL INTERFACE

studied extensively in view of beir

ubiquitous occurrence in chemistry, biology and various technological by solvent fluctution, according to belebred theortical fromew developed by Rudolph A. Marcus. Electron transfer processes have also been studied in the case of water-oil and molecular dynamics.

In this context, Prof Morita and his collaborators have studied the electron transfer reaction between the molecul ferrocene and the anion ferricyanide at a water-oil interface. The use of the multidimensional free energy approach has in this case allowed them to resolve a long-standing and crucial issue in understanding the mechanism of electron transfer between the two redox complexes, i.e. complexes with the themselves or with other substrates. Using a generalised coordinate donor-acceptor distance and solve fluctuations, they were able to prove th a heterogeneous mechanism, in which the donor and acceptor complexes are in different liquid phases when electron is transferred between them, is preferred over the homogenous route, with the two complexes in the same phase. Their simulation results are again in excellent agreement with available electrochemical measurements.

\section{LIGAND-ASSISTED ION}

RANSPORT: THE SHUTTLING MECHANISM

Small, hydrophilic ions like F-are usually dificult to transport across presence of specific counterions, even in trace amounts, is often sufficient to dramatically enhance their transport rates. For instance, traces of the tetrahexyl ammonium cation, $\mathrm{N}\left(\mathrm{C}_{6} \mathrm{H}_{13}\right)_{4}^{+}$, are known to render the $\mathrm{F}^{-}$transport facile, whereas the corresponding tetrabutyl cation $\mathrm{N}\left(\mathrm{C}_{4} \mathrm{H}_{9}\right)_{4}{ }^{+}$has no effect. There must therefore exist a catalytic mechanism promoting $\mathrm{F}$ transport that is active in the presence of the former cation, but not in the presence of the latte By carrying out a series of molecular dynamics simulations of $F$ - migration through a water-oil interface in the field (mimicking conditions typica ( Prof Morita has shown that some, counterions can form transient complexes with the F-ions and assist them as they cross the interface.

With the aid of the multidimensional free energy approach, he has been able to identify two competing transport paths and to define a bifurcation criterion that makes it possible to predict whether a transient complex can form in the presence of a specific ligand, and, therefore, whether this ligand can be used to promote $F$ ' transport across the interface. These resuls are important because of their potential impact in the development of efficient devices exploiting interfacial transport in a wide range of applications, including phase

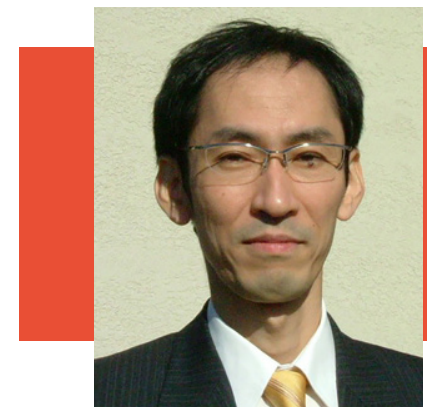

\section{Behind the Research} Prof Akihiro Morita

E: morita@tohoku.ac.jp T: $+81227957717 \quad$ W: https://comp.chem.tohoku.ac.jp

Research Objectives

Prof Morita studies the mechanisms of ion transfer at interfaces between immiscible liquids.

\section{Detail}

Akihiro Morita

Department of Chemistry

of Science

Sendai, 980-8578

Senan

Bio

Akihiro Morita received a PhD in chemistry from Kyoto University, Japan, in 1995. He worked at Kyoto University, University of Colorado, and the Institute for Molecular Science before moving to Tohoku University. His research focuses on electronic structure and molecular dynamics at liquids and interfaces, including the theory of sum frequency generation spectroscopy and mass transfer dynamics.

Funding

Japan Society for the Promotion of Science

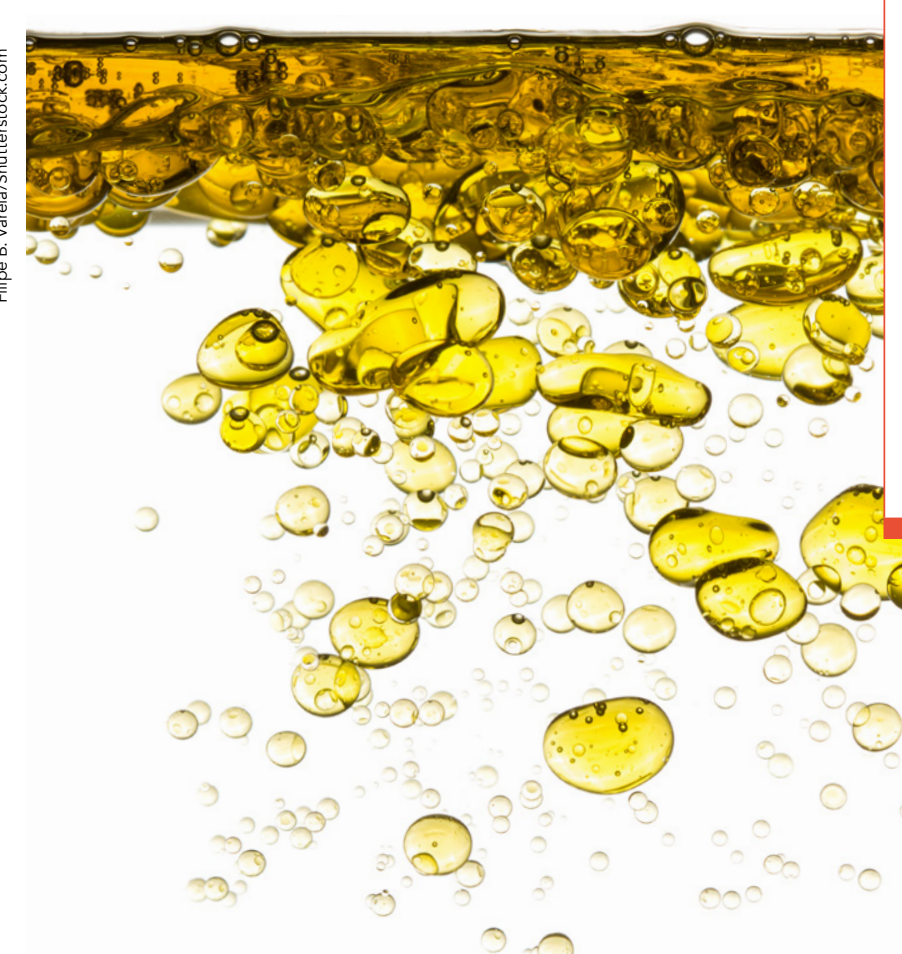

\section{References}

Koizumi, A., Tahara, H., Hirano, T., and Morita, A. (2020). Revealing Transient Shuttling Mechanism of Catalytic lon Transport through Liquid-Liquid Interface. J. Phys. Chem Lett., 11, 1584-1588.

Hirano, T. and Morita, A. (2020). Electron Transfer Mecharism at the Oi/Water Interface Revealed by Chem. B. 124, 3811-3827.

Kikkawa, N., Wang, L., and Morita, A. (2015). Microscopic Barrier Mechanism of Ion Transport through Liquid-Liquid Interface. J. Am. Chem. Soc., 137, 8022-8025.

Hellemans, A. (2015). A transient water finger slows ion transfer through water-oil interfaces. J. Am. Chem. Soc., $137,8311-8312$

\section{Personal Response}

Which academic fields will benefit most from your Work, and what further ex ansions of your approach could further enhance its applicability?

II "This work benefits the fields of physical and computational chemistry as well as other related fields of liquid interfaces, such as electroanalytical chemistry, interfaces, and chemical engineering of separation using membranes.

The present approach is quite powerful to reveal the molecules at interaces, though spatial scale is rather spatial scale, we are working to cos in a compreher dynamics simulation with the mesoscopic continum model. The multiscale approach will extend the applicability beyond the usual molecular simulation to incorporate mesoscopic scale of diffuse double layer in
a unified viewpoint."

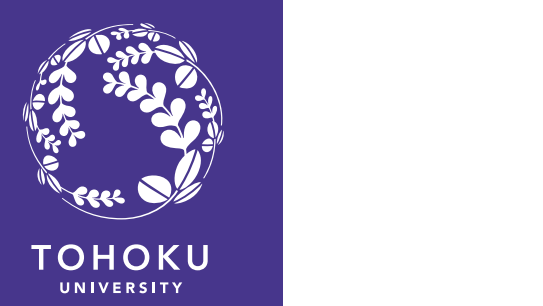

